\title{
A MINIMAL STIMULATION PROTOCOL WITH SIMPLIFIED MONITORING FOR SUPEROVULATION AND INTRAUTERINE INSEMINATION
}

\author{
Wafaá Moustafa Aboul Enien, M.D. \\ Department of Obstetrics and Gynaecology, Shatby Hospital for Women, \\ Faculty of Medicine, Alexandria University
}

\section{ABSTRACT}

Objectives: To evaluate the efficacy and cost of a simplified superovulation regimen in infertile patients undergoing intrauterine insemination (IUI).

Design : Analysis of 170 minimal stimulation and 150 conventional HMG cycles.

Setting : Infertility Clinic, Shatby Hospital for Women, Alexandria.

Patients : 180 women with either unexplained infertility or ovulatory dysfunction who ovulated with clomiphene citrate but failed to conceive.

Intervention : 90 patients underwent IUI using a simplified superovulation regimen that included clomiphene citrate $100 \mathrm{mg}$ on cycle days 3 through 7 and HMG $75 \mathrm{IU}$ on cycle days 5, 7, 9 and 11 . A control group ( $\mathrm{n}=90$ ) received the standard HMG protocol. In both groups many patients received more than one treatment cycle.

Main outcome measures: Clinical pregnancy rate, medication and monitoring costs.

Results : There was no difference in the couples of the two stimulation protocols regarding age, duration and type of infertility, as well as, cause of infertility. The monitoring costs and total ampoules of HMG required differed significantly (4.0 ampoules for minimal stimulation versus $14 \pm 3.2$ in the conventional HMG). The clinical pregnancy rates per cycle were comparable ( $7.78 \%$ for minimal stimulation versus $11.11 \%$ for HMG). No patients were admitted for ovarian hyperstimulation.

Conclusion : Minimal stimulation for IUI is less expensive than conventional HMG stimulation and minimizes monitoring and patient discomfort. The comparable pregnancy rates and decreased medication costs of minimal stimulation justifies further evaluation of its role in the treatment of infertility.

Keywords : Infertility, clomiphene citrate, HMG, intrauterine insemination, pregnancy rate, minimal stimulation.

\section{INTRODUCTION}

Infertility is said to be unexplained when a couple fails to conceive and no definite cause can be diagnosed after a complete check-up. The prevalence of unexplained infertility ranges from $6 \%$ to $60 \%$ with an average of $20 \%$. $^{(1)}$ Treatment of unexplained infertility is generally empirical aiming at the release of additional oocytes creating more opportunities for conception or correction of subtle less predictable causes of ovulatory dysfunction ${ }^{(2)}$.

Clomiphene citrate (CC) is an inexpensive medication that can affect a mild superovulation in patients with an intact hypothalamic-pituitaryovarian axis. It is used as the initial agent for ovarian stimulation because of its economical nature and ease of administration ${ }^{(3)}$.

In patients failing to conceive after four to six ovulatory cycles with $\mathrm{CC}$, switching to controlled ovarian hyperstimulation $(\mathrm{COH})$ with human 
menopausal gonadotrophin (HMG) and intrauterine insemination (IUI) offers an effective and appropriate alternative to assisted reproductive technologies $(\mathrm{ART})^{(4)}$. With reported success rates of $10-15 \%$ per cycle, COH/IUI has become a cost effective treatment compared with ART while being much less complex and invasive.

However, the drawbacks of HMG include the escalating cost of a treatment cycle in view of the medication costs, the necessity for increased monitoring by hormone assays and ultrasound, frequent injections, emotional stress on patients, as well as, pregnancy complications relating to high rate of pregnancy loss and multiple gestation ${ }^{(5,6)}$.

Treatment regimens using $\mathrm{CC}$ combined with gonadotrophins have been devised to decrease costs by reducing the total amount of HMG necessary in an ovarian stimulation cycle, without sacrificing effectiveness ${ }^{(7)}$.

Minimal stimulation protocols have been described for ART resulting in clinical pregnancy rates comparable to full HMG stimulation but at a much lower expense ${ }^{(8,9)}$. Moreover, in a study by Dhaliwal et al(10) minimal stimulation appeared to be effective in cases of unexplained infertility undergoing IUI.

The decreased medication costs and reduced time commitment led to a high patient acceptance of this approach ${ }^{(11)}$.

The main aim of this study was to evaluate the efficacy and cost of a simplified superovulation regimen in infertile patients undergoing intrauterine insemination.

\section{MATERIALS \& METHODS}

\section{Patient groups}

The present study was conducted in the
Infertility Clinic of the Department of Obstetrics and Gynaecology, Shatby University Hospital for Women, Alexandria.

Infertility work-up was carried out according to the World Health Organization Protocol for standardized investigation of infertile couples ${ }^{(12)}$. 180 women with either unexplained infertility $(n=103)$ or ovulatory dysfunction $(n=77)$ who ovulated with clomiphene citrate but failed to conceive were included in the study. Patients were offered either a simplified superovulation regimen combining $\mathrm{CC}$ and HMG or the conventional HMG protocol along with IUI.

Eligibility criteria included age of 20-35 yrs, serum day $3 \mathrm{FSH}$ levels $\leq 10 \mathrm{mIU} / \mathrm{ml}$, normal TSH and prolactin levels, normal HSG and/or laparoscopy, normal semen parameters and an intact hypothalamic-pituitary ovarian axis as determined by menstrual history or withdrawal bleeding after a progestin challenge.

\section{Ovarian stimulation protocols}

All patients received a baseline transvaginal ultrasound examination on cycle day 1 or day 2 . Serum levels of LH, FSH and $E_{2}$ were determined on cycle day 3 . If there were no ovarian cysts or elevations of $E_{2}>70 \mathrm{pg} / \mathrm{ml}$, ovarian stimulation was started.

Patients were divided randomly into two groups. Group A included 90 women who were offered clomiphene citrate $100 \mathrm{mg} /$ day orally during cycle days 3 through 7 . Higher doses were selected based on previous individual responses to clomiphene citrate. The objective being to administer a dose that produced two or more large follicles. Concurrently, HMG 75 IU was administered intramuscularly on days 5,7,9 and 11 of the cycle to help in the late follicular growth of 
dominant follicles recruited with clomiphene citrate and for better development of the endometrium.

On cycle day 12 , transvaginal ultrasonography was performed to assess follicle development. In general, one or two sonograms were performed and the peak follicle diameter was recorded or projected assuming $1-2 \mathrm{~mm}$ increase in the mean diameter of the leading follicle/day. The criteria for triggering ovulation were the presence of at least one follicle with a mean diameter greater than or equal to $18 \mathrm{~mm}$. The majority of patients had reached adequate follicular maturity by cycle day 13 and ovulation was triggered with $10,000 \mathrm{IU}$ of HCG administered intramuscularly.

For comparison, group B ( $n=90)$ received the conventional HMG protocol (controls). Both groups were matched for age, diagnosis and date of stimulation.

Patients received daily doses of HMG starting with $150 \mathrm{IU} /$ day unless the patients had exhibited unusual sensitivity or tolerance to gonadotrophins. This was individualized based on patients' previous response to ovarian stimulation therapy (clomiphene citrate, minimal stimulation, and/or previous HMG). Adjustments to HMG dosage and timing of HCG administration were determined by serial ultrasound monitoring and serum oestradiol determinations commenced on day 10 then afterwards on alternate days.

HCG (10,000 IU) was given under the same guidelines as described for the minimal stimulation protocol. In both groups, intrauterine insemination of husband's semen was performed 36-40 hrs after HCG administration.

\section{Semen preparation and insemination technique}

Couples were advised abstinence for 3 days.
Semen was collected in sterile containers and was allowed to liquefy at $37^{\circ} \mathrm{C}$ then prepared by wash and swim-up technique using Ham's F-10 culture medium. The washed pellet obtained was diluted in a final volume of $0.5 \mathrm{ml}$ of Ham's F-10 and injected into the uterine cavity with an IUI catheter (Gynétics Medical Products, N.V. Hamont- Achel, Belgium).

All patients received luteal support beginning from the day of IUI in the form of vaginal progesterone using micronised progesterone capsules (100 mg, 3 times a day; utrogestan: Besins-International, S.A-France). Luteal support was administered until menstruation or positive pregnancy test.

\section{Monitoring of outcome}

Pregnancy was tested 4-5 days after the missed period followed by ultrasound examination 2 weeks later for the detection of gestational sac and cardiac activity.

The incidence of miscarriage, multiple pregnancies and ovarian hyperstimulation syndrome (OHSS) was assessed in both protocols. Moreover, the costs of monitoring, CC, HMG and HCG were also assessed.

\section{Statistical analysis}

The data were collected, entered into an IBM compatible computer using SPSS then checked for accuracy and normality. Quantitative data were presented as $\mathrm{X} \pm \mathrm{SD}$, whereas qualitative data were presented as number and percentages. The students' t-test was used for comparison between two means and the Z-test for comparison between two percentages. The Mann-Whitney test was used as t-test for number of motile sperms inseminated as it was not normally distributed. Throughout the 
analyses, $\mathrm{p}<0.05$ defined the level of statistical significance.

\section{RESULTS}

Hundred and eighty infertile couples underwent 320 ovarian stimulation cycles along with IUI and resulted in 31 conceptions. Ninety women (group A) completed 170 cycles of minimal stimulation and the remaining 90 women (group B) completed 150 HMG cycles. There were no differences between the two groups regarding the mean age, years of infertility, type of infertility or the indications for treatment. Infertility characteristics and diagnoses in the two stimulation protocols are shown in table 1 .

Among the 90 patients who received minimal stimulation for 170 cycles, the mean number of dominant follicles per cycle was $1.64 \pm 0.69$ whereas the mean number of dominant follicles in the 150 treatment cycles received by the controls was $2.68 \pm 1.30$. The difference was statistically significant $(p<0.001)$. The number of ampoules of HMG used in the two treatment groups was significantly different ( 4 ampoules for group $A$ and $14 \pm 3.2$ ampoules for group $B \mathrm{p}<0.001$ ). Follicle monitoring in minimal stimulation required one to two visits for transvaginal ultrasound while three to four visits were required by subjects receiving the full HMG protocol.

The pregnancy rate per cycle, as well as, per couple did not differ significantly between the two stimulation protocols $(7.78 \%$ and $15.56 \%$ for minimal stimulation versus $11.11 \%$ and $18.89 \%$ for full HMG stimulation). Miscarriage rate was significantly higher in women who received the full HMG protocol $(21.11 \%$ versus $6.67 \%$ in minimal stimulation $\mathrm{p}<0.05$ ). None of the women in the minimal stimulation group had multiple gestation or ovarian hyperstimulation. The data are shown in table II.

On comparing the pregnant and non pregnant groups in both protocols, there was no significant difference in the age of the female partner, length of infertility or total number of motile sperms inseminated. On the contrary, patients who conceived had significantly more follicles greater than $10 \mathrm{~mm}$ and more follicles greater than $16 \mathrm{~mm}$ (Table III and Table IV). Furthermore, in the conventional HMG protocol, the peak oestradiol concentration on the day of HCG administration was significantly higher in pregnant compared to non-pregnant females (Table IV).

Comparing the medication and monitoring costs in both protocols, the current market rate of clomiphene citrate $100 \mathrm{mg}$ daily for 5 days is 8.75 LE, 1 ampoule of HMG (75 IU FSH, 75 IU LH) costs approximately 50 L.E., 5,000 IU of HCG costs 15 L.E. The hospital charges are 40 L.E. for one transvaginal sonographic assessment and 30 L.E. for one serum oestradiol assay. Based on these figures, the minimal stimulation expenses of medication and monitoring are approximately one third of the full HMG stimulation per cycle. (Table V)

\section{DISCUSSION}

Anovulation in women desiring fertility is the most common indication of ovulation induction. However, ovarian stimulation may be also recommended for women who apparently have spontaneous ovulation ${ }^{(2)}$.

Controlled ovarian hyperstimulation with HMG in conjunction with IUI has been advocated for improving fecundity in patients with unexplained infertility and in those who fail to conceive with 
C.C.. Induction protocols that require lower dosages of HMG are attractive due to reduced time commitment, cost and minimal monitoring ${ }^{(7)}$.

In the present study, the pregnancy rate per couple, as well as, per cycle did not differ significantly between the minimal and full HMG protocols. Similar observations were made by previous investigators $(13,14)$. Monitoring patients in the conventional HMG group required several transvaginal sonographic assessments and serum $\mathrm{E}_{2}$ determinations whereas a single visit on cycle day 12 sufficed in the minimal stimulation group. If the lead follicle was not at least $18 \mathrm{~mm}$ in diameter on the day of ultrasound, the timing of HCG administration was projected by assuming a follicular growth rate of approximately 1-2 mm per day ${ }^{(15)}$. This usually resulted in HCG being given within 3 days (between days 13-15 of the cycle).

Rarely, a second ultrasound was performed if the lead follicle diameter was $<14 \mathrm{~mm}$ in order to properly time the ovulation inducing dose of HCG. Patients without follicular development of $\geq 16 \mathrm{~mm}$ by cycle day 15 were considered treatment failures and advised to proceed with HMG therapy.

In the current study, all pregnancies in the minimal stimulation group and $94.87 \%$ in the conventional HMG group were singleton. Pregnancies following HMG stimulation have been reported to have an increased risk of spontaneous miscarriage ranging from $12-31 \%(6)$. The miscarriage rate in the current study was $6.67 \%$ in minimal stimulation compared to $21.11 \%$ in the full stimulation group. This may be explained by the fact that patients who undergo full stimulation have mean post-HCG $\mathrm{E}_{2}$ levels in excess of twice that of the minimal stimulation protocol.
Hyperestrogenicity may alter the balance of $E_{2}$ and $P$ and lead to inhibition of embryonic implantation $(16,17)$

In a study by Vargyas et al, ${ }^{(18)}$ ovarian stimulation for ART using C.C. concomitant with multiple injections of HMG yielded significantly fewer oocytes compared to full stimulation, yet a higher percentage of mature oocytes were produced. It is suggested that C.C. initiates optimal follicle selection and minimal HMG support may be required to achieve late and continued follicular growth.

However, since CC is an anti-oestrogen, there is a possibility that it may adversely affect endometrial receptivity ${ }^{(19,20)}$. Subtle defects may also occur including luteal phase defect or altered tubal function ${ }^{(21)}$. Other reports do not confirm these findings ${ }^{(22)}$.

Hence, patients with repetitive thin endometrium or those who fail to conceive after 4 cycles of CC/HMG/IUI, should be offered treatment with HMG/IUI before proceeding to ART.

In the present study, the simplified superovulation regimen was easy to administer, required a single monitoring session, smaller amounts of gonadotrophins, lower miscarriage rate and virtually eliminated the risk of OHSS. The comparable pregnancy rates and decreased medication costs of minimal stimulation justifies further evaluation of its role in the treatment of infertility. This approach provides an alternative for those patients with unexplained infertility and C.C. failure who cannot afford conventional regimens or who are concerned with exposure to high dosages of fertility medications. 


\section{REFERENCES}

1- Coulam CB, Moore SB, O'Fallon W. Investigating unexplained infertility. Am J Obstet Gynecol 1988; 158: 1374-1381.

2- Stovall DW, Guzick DS. Current management of unexplained infertility. Curr Opin Obstet Gynecol $1993 ; 5: 228-233$.

3- Glazener CM, Coulson C, Lambert PA, Watt EM, Hinton RA, Kelly NG et al. Clomiphene treatment for women with unexplained infertility: placebo-controlled study of hormonal responses and conception rates. Gynecol Endocrinol 1990; 4: 75-83.

4- Dodson WC, Whitsides DB, Hughes CLJr, Easley HA, Haney AE. Superovulation with intrauterine insemination in the treatment of infertility: A possible alternative to gamete intrafallopian transfer and in vitro fertilization. Fertil Steril 1987; 48: $441-445$.

5. Nulsen JC, Walsh S, Dunez S, Metzger DA. A randomized and longitudinal study of human menopausal gonadotrophin with intrauterine insemination in the treatment of infertility. Obstet Gynecol 1993; 82: 780-786.

6- Ben Rafael Z, Dor J, Mashiach S et al. Abortion rate in pregnancies following ovulation induced by human menopausal gonadotrophin/human chorionic gonadotrophin. Fertil Steril 1983; 39: 157-161.

7- Benadiva CA, Davis O, Kligman I, Liu HC, Rosenwaks Z. Clomiphene citrate and HMG: an alternative stimulation protocol for selected failed in vitro fertilization patients. J Assist Reprod Genet $1995 ; 12: 8-12$.

8- Corfman RS, Milad MP, Bellavance TL, Ory SJ, Erickson LD, Ball GD. A novel ovarian stimulation protocol for use with the assisted reproductive technologies. Fertil Steril 1993; 60: 864-870.

9- De Vane GW, Gangrade BK, Wilson, R, Loy RA. Optimal pregnancy outcome in a minimal stimulation in vitro fertilization programme. Am J
Obstet Gynecol 2000 Aug; 183(2): 309-313.

10- Dhaliwal LK, Sialy RK, Gopalan S, Majumdar S. Minimal stimulation protocol for use with intrauterine insemination in the treatment of infertility. J Obstet Gynecol Res 2000 Dec; 28(6): 295-299.

11- Hurst BS, Tucker KE, Schlaff WD. A minimally monitored assisted reproduction stimulation protocol reduces cost without compromising success. Fertil Steril 2002; 77(1): 98-100.

12- World Health Organization Manual for Simplified Management of Infertile Couple. WHO special programme of research and development and research training in human reproduction. Geneva; WHO: 1986.

13- Lu RY, Chen AL, Atkinson EJ, Lee SH, Erickson LD, Ory SJ. Minimal stimulation achieves pregnancy rates comparable to human menopausal gonadotrophins in the treatment of infertility. Fertil Steril 1996 Mar; 65(3): 583-587.

14- Williams RS, Kipersztok S, Hills D, Dattilo M. A novel, simplified and cost effective protocol for superovulation and intrauterine insemination. J Fla Med Assoc 1997 Jan-Jul; 84(5): 316-319.

15- Gougeon A. Dynamics of follicular growth in the human: a model from preliminary results. Hum Reprod 1986; 1: 81-87.

16- Maclin VM, Radwanska E, Binor Z, Dmowski WP. Progesterone; oestradiol ratios at implantation in ongoing pregnancies, abortions, and non conception cycles resulting from ovulation induction. Fertil Steril 1990; 54: 238-244.

17- Paulson RJ, Sauer MV, Lobo RA. Embryo implantation after human in vitro fertilization: importance of endometrial receptivity. Fertil Steril 1990; 53: 870-874

18- Vargyas JM, Morente C, Shangold G, Marrs RP. The effect of different methods of ovarian stimulation for human in vitro fertilization and embryo replacement. Fertil. Steril 1984; 42: $745-749$. 
19- Rogers PAW, Milne BJ, Trounson AO. A model to show human uterine receptivity and embryo viability following ovarian stimulation for vitro fertilization. J In Vitro Fert Embryo Transt 1986; 3: 93-98.

20- Nelson LM, Hershlad A, Kurl RS, Hall JL, Stillman RJ. Clomiphene citrate directly impairs endometrial receptivity in the mouse. Fertil Steril 1990; 53: $727-731$
21- Cook CI, Schroeder JA, Yussman MA, Sanfilippo JS. Induction of luteal phase defect with clomiphene citrate. Am J Obstet Gynecol 1984; 149: 613-616.

22- Thatcher SS. Donachie KM, Glasier A, Hillier SG, Baird DT. The effect of clomiphene citrate on the histology of the human endometrium in regularly cycling women undergoing in vitro fertilization. Fertil Steril 1988; 49:296-301.

Table I : Infertility characteristics and diagnoses

\begin{tabular}{|l|c|c|}
\hline & $\begin{array}{c}\text { Minimal stimulation } \\
\mathbf{n}=90\end{array}$ & $\begin{array}{c}\text { Full stimulation } \\
\mathbf{n}=90\end{array}$ \\
\hline Average age (years) & $33.21 \pm 4.53 \cdot$ & $34.21 \pm 7.27 \cdot$ \\
\hline Length of infertility (years) & $2.82 \pm 3.94 \cdot$ & $3.91 \pm 4.20 \cdot$ \\
\hline Primary infertility (\%) & $52.22(47 / 90)$ & $61.11(55 / 90)$ \\
\hline Diagnosis & & 47.78 \\
Ovulatory dysfunction \% & 35.56 & 51.11 \\
Uniexplained infertility \% & 63.33 & \\
\hline
\end{tabular}

All non significant

- Values are mcan \pm S.D.

Table II : Outcome of treatment with both protocols

\begin{tabular}{|l|c|c|}
\hline & $\begin{array}{c}\text { Minimal stimulation } \\
\mathbf{n}=90\end{array}$ & $\begin{array}{c}\text { Fuil stimulation } \\
\mathbf{n}=90\end{array}$ \\
\hline Mean number of treatment cycles & $170(1.89 \pm 0.32$ & $150(1.67 \pm 0.47)$ \\
\hline No. of dominant follicles & $1.64 \pm 0.69$ & $2.68 \pm 1.30^{*}$ \\
\hline No. of monitoring visits & $1.3 \pm 0.61$ & $3.15 \pm 1.21 *$ \\
\hline No. of HMG ampoules & 4 & $14 \pm 3.2^{*}$ \\
\hline Pregnancy rate/cycle & $7.78 \%$ & $11.11 \%$ \\
\hline Pregnancy rate/couple & $15.56 \%(14 / 90)$ & $18.89 \%(17 / 90)$ \\
\hline Miscarriage \% & $6.67(6.90)$ & $21.11 \%(19 / 90)^{*}$ \\
\hline Multiple pregnancy \% & - & $5.13 \%$ \\
\hline Ovarian hyperstimulation & & - \\
\hline syndrome (OHSS) & & \\
\hline
\end{tabular}

* Significant $(p<0.001)$ 
Table III : Characteristics of pregnant and non-pregnant cycles (minimal stimulation protocol)

\begin{tabular}{|l|c|c|}
\hline & $\begin{array}{c}\text { Pregnant } \\
\mathbf{n = 1 4}\end{array}$ & $\begin{array}{c}\text { Non-pregnant } \\
\mathbf{n}=76\end{array}$ \\
\hline Age $(\mathrm{yrs})$ & $32.16 \pm 3.39$ & $33.4 \pm 4.7$ \\
\hline Length of infertility (yrs) & $2.46 \pm 1.90$ & $2.89 \pm 4.19$ \\
\hline Follicles $>10 \mathrm{~mm}$ & $5.72 \pm 2.18$ & $4.32 \pm 2.13 *$ \\
\hline Follicles $>16 \mathrm{~mm}$ & $2.34 \pm 1.2$ & $1.51 \pm 1.4 *$ \\
\hline $\begin{array}{l}\text { Total number of motile sperms } \\
\text { inseminated }\left(10^{6}\right)\end{array}$ & $36.63 \pm 22.43$ & $45.84 \pm 52.97$ \\
\hline
\end{tabular}

* Significant $(\mathrm{p}<0.05)$

Table IV : Characteristics of pregnant and non-pregnant cycles (full stimulation protocol)

\begin{tabular}{|l|c|c|}
\hline & $\begin{array}{c}\text { Pregnant } \\
\mathbf{n}=\mathbf{1 7}\end{array}$ & $\begin{array}{c}\text { Non-pregnant } \\
\mathbf{n}=73\end{array}$ \\
\hline Age $(\mathrm{yrs})$ & $33.4 \pm 2.27$ & $34.4 \pm 4.60$ \\
\hline Length of infertility $(\mathrm{yrs})$ & $3.72 \pm 1.3$ & $3.96 \pm 2.18$ \\
\hline Follicles $>10 \mathrm{~mm}$ & $8.36 \pm 1.9$ & $7.32 \pm 1.7 *$ \\
\hline Follicles $>16 \mathrm{~mm}$ & $3.95 \pm 2.6$ & $2.61 \pm 1.6^{*}$ \\
\hline Peak $\mathrm{E}_{2}(\mathrm{pg} / \mathrm{ml})$ & $1750 \pm 311$ & $1.600 \pm 269^{*}$ \\
\hline $\begin{array}{l}\text { Total number of motile sperms } \\
\text { inseminated }\left(10^{6}\right)\end{array}$ & $42.76 \pm 21.3$ & $34.36 \pm 51.6$ \\
\hline
\end{tabular}

* Significant $(p<0.05)$

Table V : Medication and monitoring costs per cycle

\begin{tabular}{|c|c|c|}
\hline 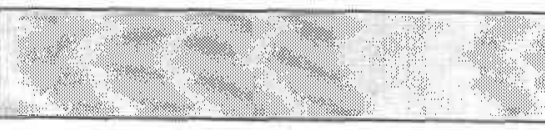 & Minimal stimulation & Full stimulation \\
\hline C.C (10 tablets) & 8.75 L.E. & $w_{n=5}-$ \\
\hline HMG (amp = 50 L.E.) & 4 amp $=200$ L.E. & $14 \mathrm{amp}=700 \mathrm{~L} . \mathrm{E}$. \\
\hline HCG (amp = 15 L.E.) & $2 \mathrm{amp}=30 \mathrm{~L} \cdot \mathrm{E}$. & $2 \operatorname{amp}=30 \mathrm{~L} E$ \\
\hline Ultrasound ( 1 study = 40 L.E.) & 40 L.E. & 120L.E. \\
\hline $\mathrm{E}_{2}$ assay $(1$ test $=30 \mathrm{~L} . \mathrm{E})$. & - & $120 \mathrm{~L}: \mathrm{E}$. \\
\hline Luteal progesterone & 38.50 L.E. & 38.50 L.E. \\
\hline Total & 317.25 L.E. & $1008.50 \mathrm{LE}, *$ \\
\hline
\end{tabular}

* Significant $(\mathrm{p}<0.05)$ 